\title{
étude expérimentale et modélisation numérique de vibrations induites dans un tunnel de métro
}

\author{
F. CHAPEL \\ Chef de Travaux, Ecole Centrale des Arts et Manufactures* \\ D. LEFEBVRE \\ Chercheur, Ecole Centrale des Arts et Manufactures*
}

\section{AVANT-PROPOS}

Le réseau ferré de la R.A.T.P. comprend $250 \mathrm{~km}$ de lignes souterraines, implantées à proximité des immeubles riverains. La circulation des trains provoque des vibrations qui se transmettent à travers le sol, accompagnées de grondements sourds qui sont d'autant plus sensibles que l'exploitation des lignes ne s'arrête que pendant quatre heures trente la nuit. Afin de mieux lutter contre les nuisances, la R.A.T.P. a souhaité pouvoir disposer d'un outil lui permettant de mieux connaître les phénomènes de propagation en fonction de la nature des sols. Elle a fait appel au Laboratoire de Mécanique des Sols-Structures du centre de recherche de l'Ecole Centrale des Arts et Manufactures et, sous la direction du Professeur BIAREZ, une équipe de recherche a été formée, animée par MM. CHAPEL et LEFEBVRE. Ainsi a vu le jour une nouvelle application de méthodes numériques à l'analyse des phénomènes vibratoires dans les sols avec pour objectif final l'amélioration de la qualité de la vie de centaines de riverains.

\footnotetext{
* 92290 Chatenay-Malabry.
}

\section{INTRODUCTION}

Le problème des vibrations induites par les rames de métro dans le sol et les immeubles riverains était ordinairement abordé au cas par cas, de façon empirique. Le développement récent des méthodes numériques appliquées à la mécanique des milieux continus nous a permis d'envisager une approche différente, s'inscrivant dans une méthodologie plus générale, qui est celle de la mécanique des milieux continus appliquée aux sols.

Nous avons ainsi eu recours à un modèle de calcul dans lequel les matériaux en présence (sol, béton...) sont supposés être des matériaux continus, dotés chacun d'une loi rhéologique, soumis à des efforts extérieurs (passage du train) et à des conditions aux limites bien définies. Ce modèle pourra servir d'outil prévisionnel et curatif des vibrations dues au métro.

Les recherches ayant conduit à la mise au point et à la validation numérique et expérimentale de ce modèle ont été réalisées grâce au concours actif de la R.A.T.P., Direction des Services Techniques, Service des Etudes, Division Environnement et Confort. 
Nous remercions particulièrement Madame Lizerand ainsi que Messieurs Flahaut et Colline pour l'appui constant qu'ils nous ont prodigué tout au long de cette étude.

\section{MÉTHODOLOGIE DE RECHERCHE}

Pour cela, les équations fondamentales de la dynamique ont été résolues à l'aide d'un code de calcul Eléments Finis dont le principe est détaillé plus loin $(\S I .4)$ et pour lequel une loi de comportement viscoélastique linéaire a été adoptée.

\subsection{Principe de validation d'un code de calcul par des essais in situ}

L'exploitation industrielle ultérieure du code de calcul est subordonnée à sa fiabilité par rapport à la réalité in situ. D'un point de vue fondamental, cette fiabilité s'appuie sur:

- la validation de l'hypothèse de la visco-élasticité linéaire, fondée elle-même sur l'hypothèse de petites déformations :

- notre capacité à estimer les valeurs des paramètres d'entrée du modèle de calcul, que l'on peut ranger en trois classes:

- les propriétés mécaniques du sol et des matériaux du tunnel;

- la géométrie du système tunnel/sol;

- l'excitation à la source (à la base des rails).

Nous nous sommes attachés dans cette étude au deuxième point après avoir accepté l'hypothèse de comportement viscoélastique linéaire pour l'ensemble des composants du système sol-tunnel. Pour l'expliciter, nous avons été conduits à concevoir un plan d'essai de validation en grandeur réelle, par étapes découplant au maximum les effets des différents paramètres. Ce plan est précisé par l'organigramme de la figure 1.

Remarquons qu'un tel programme expérimental de validation, du fait du grand nombre de voies de mesure qu'il suppose (16 dans notre étude) et du volume du traitement ultérieur, doit être réalisé à l'aide d'un procédé d'acquisition P.C.M. ( Pulse Code Modulation ») compatible avec un micro-ordinateur adapté: le schéma de la chaîne d'acquisition et de traitement utilisée est donné sur la figure 2.

\subsection{Essais sismiques pour la recherche des propriétés du sol}

Les paramètres de la loi viscoélastique linéaire ont été mesurés par des essais sismiques entre sondages ( cross hole») de type différentiel (voir figure 3 ). Ces essais, fondés sur l'examen des signaux sismiques [1] se propageant à partir d'une source impulsionnelle située au sein du massif de sol, ont l'avantage:
- d'être compatibles avec les essais de laboratoire au triaxial cyclique, dans le domaine des faibles déformations $\left(\varepsilon<10^{-6}\right)[2]$, qui est bien le domaine concerné par les problèmes de nuisances;

- d'être bien adaptés aux mesures d'hétérogénéités dynamiques par couches, contrairement à tous les essais sismiques de surface (sismique-réfraction, essais "surface-sondage» ou "down hole»);

- de conduire à la connaissance des deux vitesses d'ondes $\mathrm{P}$ et $\mathrm{S}$ du milieu, donc des deux paramètres de l'élasticité linéaire $G$ (module de cisaillement) et $v$ (coefficient de Poisson), à condition:

- d'utiliser une source d'impulsion qui engendre une composante $\mathrm{S}$ repérable facilement dans les trains d'ondes,

- d'éviter, par un positionnement convenable de la source et des capteurs, l'interférence au niveau des capteurs de différents types d'ondes (réflexions et réfractions dues aux hétérogénéités),

- de constituer dans un avenir proche, un moyen de mesure de la viscosité, à condition d'analyser les signaux, non seulement en termes de temps de propagation, mais aussi en termes d'évolution de forme et de contenu fréquentiel.

Le troisième paramètre de la loi rhéologique adoptée, la viscosité, est estimé en laboratoire par mesure de l'amortissement dans l'essai triaxial cyclique.

Le dépouillement des essais sismiques conduit donc aux paramètres de la loi viscoélastique qui font partie des données à introduire dans le code de calcul.

\subsection{Code de calcul [3]}

Le problème à traiter est celui de la détermination du signal induit par un train et de son transfert dans les couches de sol jusqu'aux structures concernées par les nuisances. Grâce à l'hypothèse de linéarité des propriétés du sol, le problème sera circonscrit lorsque seront connues, dans le domaine fréquentiel, les fonctions de transfert entre la source et les points de mesure.

A partir de là, la mesure de la vibration en un point du sol peut permettre, par la résolution du problème inverse, de trouver l'excitation due au train, puis la réponse en tout point du sol. Ainsi, dans des conditions idéales, les calculs à mener pour modéliser le comportement dynamique du tunnel et du sol environnant devraient prendre en compte le caractère tridimensionnel du problème, l'interaction entre les différents composants ainsi que l'hétérogénéité du sol.

Afin de déterminer plus aisément les rôles des différents paramètres, il aurait été intéressant de pouvoir diviser l'ensemble sol-structure en sous-structures mais, au vu de l'hétérogénéité du sol, de la forme du tunnel et de la présence de sol injecté sous le radier, les composants se sont avérés trop nombreux et complexes.

Une méthode numérique directe s'est ainsi imposée: la méthode des éléments finis est adaptée à ces problèmes fortement hétérogènes puisque chaque élément, 


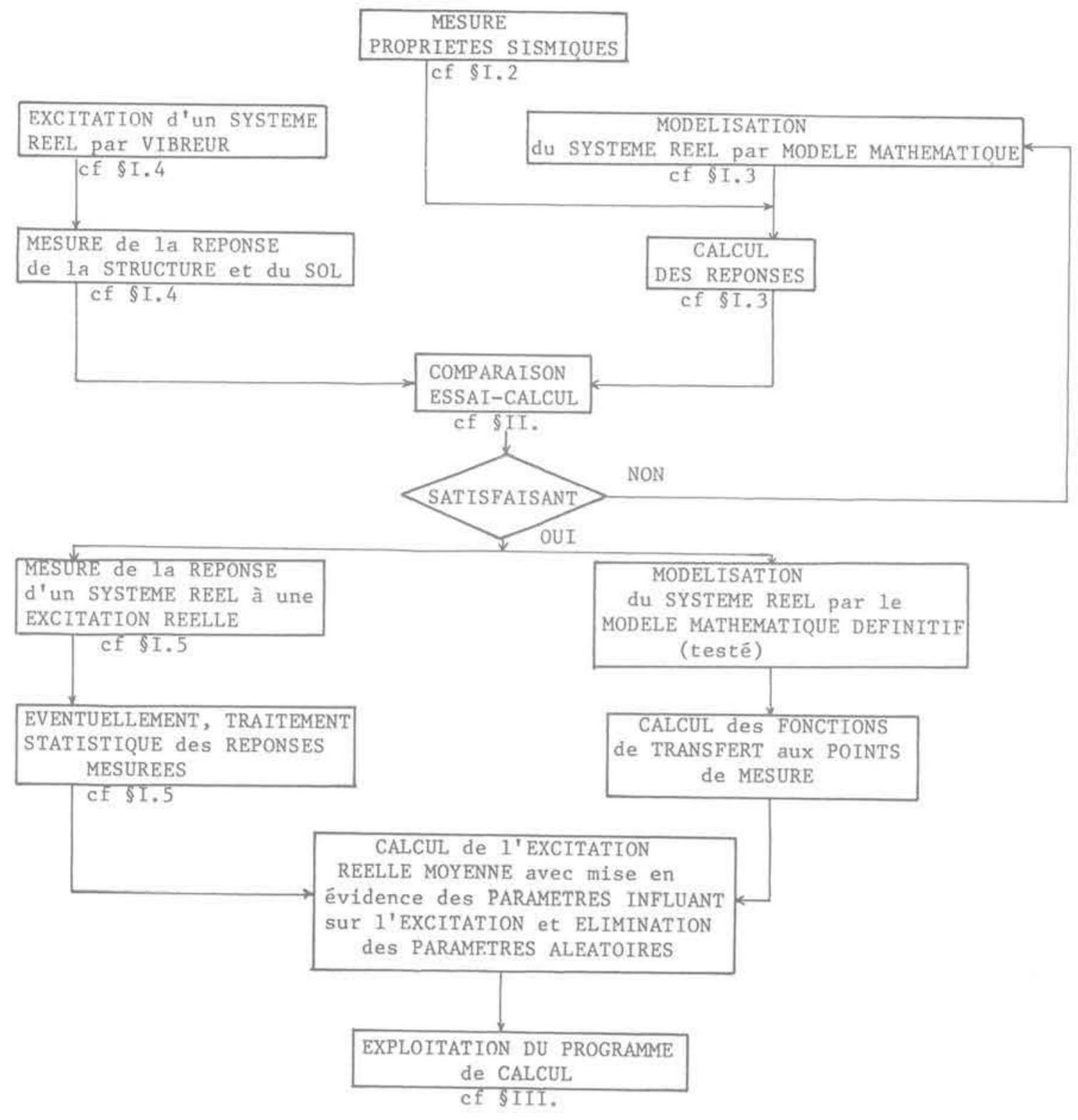

Fig. 1. - Organigramme de recherche pour la validation du code de calcul par confrontation avec un programme d'essais in situ.

en cas extrême, peut disposer de propriétés mécaniques propres.

Cependant, un calcul réellement tridimensionnel reste d'un coût prohibitif lorsque le domaine concerné est de dimension infinie dans une ou plusieurs directions.

Les calculs ont pour cette raison été menés à l'aide d'un code éléments finis bidimensionnels à déformation plane. Cette limitation importante a eu une forte incidence sur la méthodologie des essais afin que les résultats de ceux-ci puissent être comparés aux valeurs calculées. Le code de calcul résout les équations de l'élastodynamique stationnaire. Les conditions aux limites de radiation de l'énergie aux frontières artificielles sont prises en compte par des amortisseurs d'ondes P et $\mathrm{S}$ pour la frontière horizontale et par des amortisseurs neutralisant les modes propres discrétisés de vibration horizontale et verticale de la frontière latérale (frontières consistantes).

Des essais harmoniques ont été mis en œuure afin d'obtenir la validation du code de calcul et de ses paramètres d'entrée. Ils servent ensuite à la détermination des fonctions de transfert dans le sol et au calcul inverse conduisant à l'excitation clue au train.

\subsection{Essais harmoniques}

La validation expérimentale du code de calcul suppose une bonne modélisation de la sollicitation dynamique, 
Accêléromètres

piezoélectriques
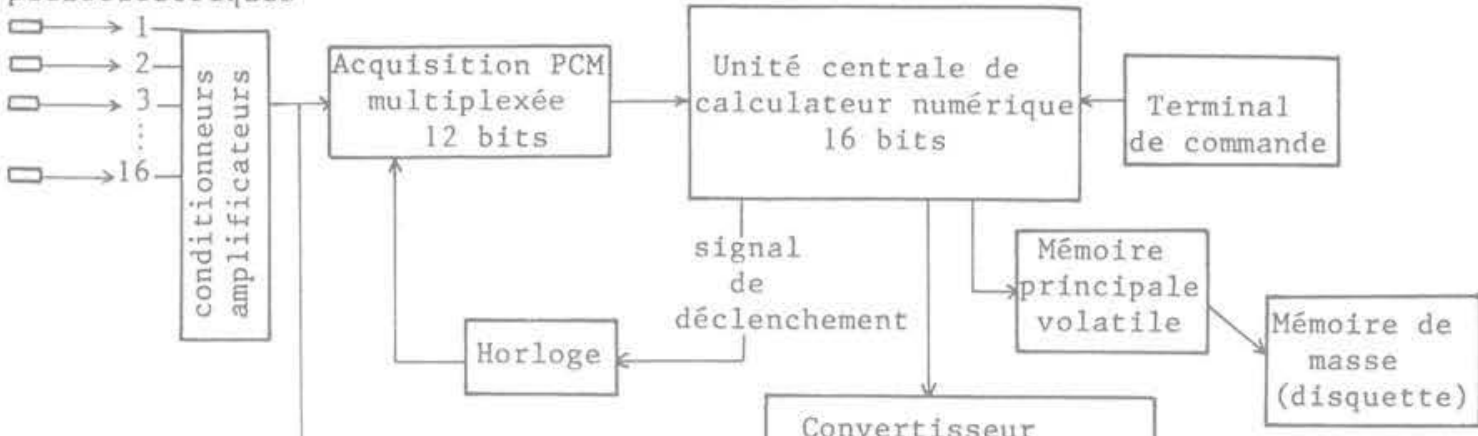

Numérique/Analogique 4 voies

Oscilloscope de contrôle

Fig. 2. - Schéma de la chaîne de saisie et de traitement des données.

Déclenchement base-temps

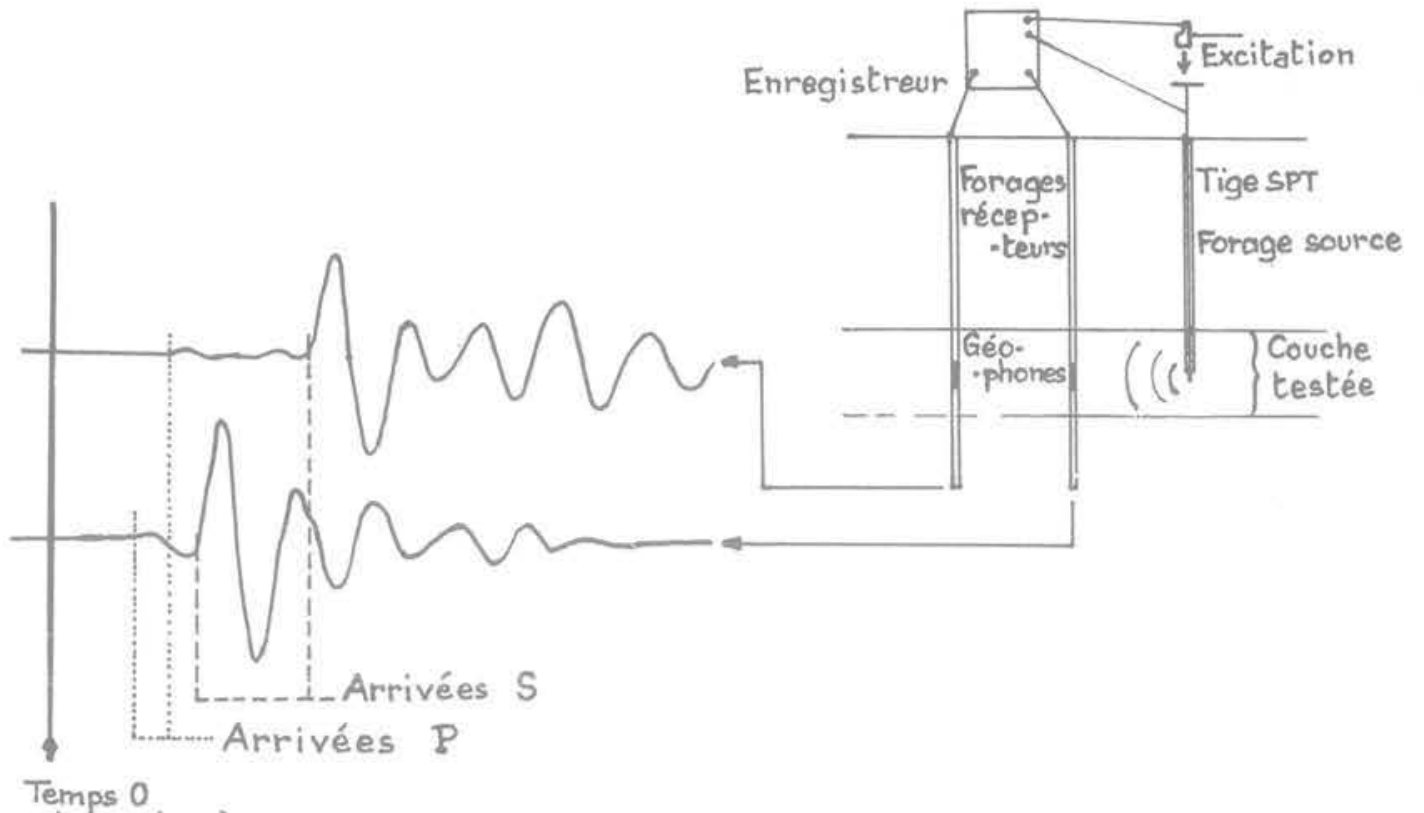

(Choc du marteau)

Fig. 3. - Enregistrement de l'essai sismique entre sondages à deux voies.

Essai réalisé dans du calcaire à Paris

à $14 \mathrm{~m}$ de profondeur.

Echelles: abscisse $2 \mathrm{~ms} /$ division

ordonnée $10 \mathrm{mV} /$ division. 


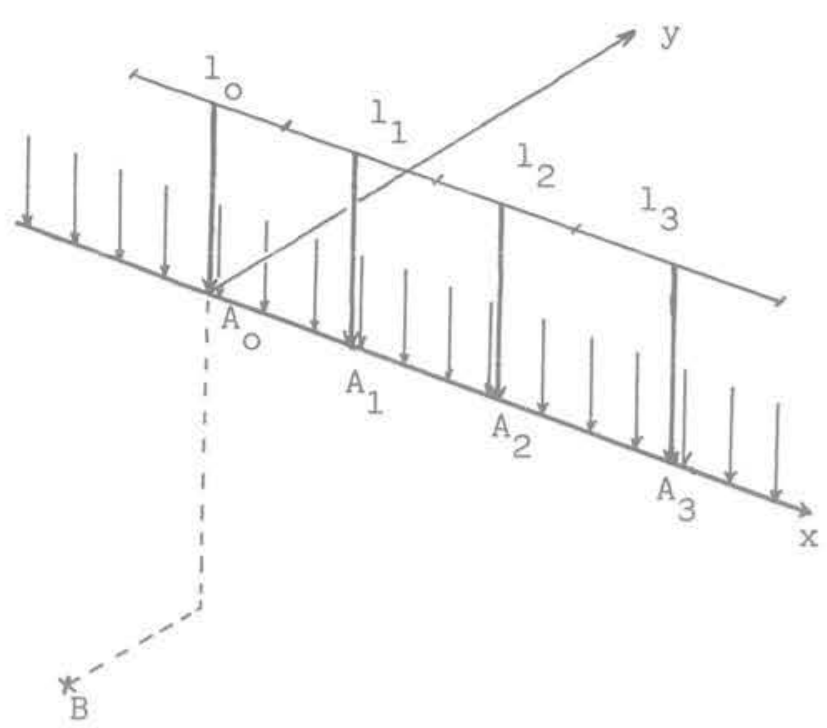

Fig. 4. - Equivalence d'une excitation linéique répartie et d'une suite discrète d'excitations ponctuelles.

donc une bonne maîtrise de l'excitation à mettre en œuvre in situ. Pour cela, nous avons eu recours à une chaîne d'excitation asservie délivrant un signal quasi ponctuel sinusoïdal de fréquence et d'amplitude pilotables. Se pose donc le problème de pouvoir reproduire correctement à l'aide de ce matériel les hypothèses de base du modèle de calcul, soit:

- L'hypothèse d'harmonicité facilement accessible et stable dans un domaine de fréquences défini par la courbe du vibreur (soit 0 à $100 \mathrm{~Hz}$ ).

- L'hypothèse de bidimensionalité avec répartition linéique continue et constante de l'excitation le long de l'axe des rails, sur le radier. Cette force répartie $\mathrm{p}=\mathrm{p}_{\mathrm{o}} \exp (\mathrm{i} \omega \mathrm{t}$ ) (fig. 4) a été reproduite expérimentalement par une suite discrète de forces quasi ponctuelles $Q_{1}$ appliquées aux points d'ancrage successifs $A_{j}$ du vibreur:

$$
Q_{\mathrm{f}}=\mathrm{P}_{\mathrm{j}} \exp \mathrm{i}\left(\omega \mathrm{t}+\psi_{\mathrm{j}}\right)
$$

oũ $\psi_{j}$ est le déphasage fictif découlant de la dérive de l'instant initial d'enregistrement au cours des différents enregistrements.

Les accélérations $\Gamma_{j}$ engendrées en un point de mesure $B$ par chaque force ponctuelle séparée $Q_{1}$ s'écrivent:

$$
\Gamma_{\mathrm{j}}=\gamma_{\mathrm{j}} \exp \mathrm{i}\left(\omega \mathrm{t}+\psi_{\mathrm{j}}+\theta_{\mathrm{j}}\right)
$$

où $\theta$ est le déphasage entre la force appliquée et l'accélération mesurée. Ces accélérations sont recomposées pour donner l'accélération linéique $\Gamma$ autour du point $\mathrm{B}$ due à la fois à la force linéique $\mathrm{p}=\mathrm{p}_{\mathrm{o}} \exp (\mathrm{i} \omega \mathrm{t})$

$$
\Gamma=\sum_{j=-\infty}^{+\infty} \frac{\gamma_{j} l_{1} \mathrm{p}_{0}}{\mathrm{P}_{j}} \exp \mathrm{i}\left(\omega \mathrm{t}+\theta_{\mathrm{j}}\right)
$$

grandeur véritablement calculée par le modèle de calcul bidimensionnel à déformation plane.

Dans la pratique, la contribution de $A_{1}$ devient négligeable devant la contribution cumulée de $A_{0}, A_{1} \ldots$, $\mathrm{A}_{\mathrm{j}-1}$ lorsque $\mathrm{j}>3$ avec $\mathrm{l}=4 \mathrm{~m}$.

\subsection{Excitation due au passage d'un train}

Les tests expérimentaux de fiabilité du code de calcul étant réalisés, il reste à définir la méthode d'estimation de l'excitation réelle due au passage d'un train. Pour cela, on observe en un point B situé à proximité du tunnel l'évolution au cours du temps de l'accélération ponctuelle $\gamma(\mathrm{t})$.

Pour utiliser cette information dans la recherche de l'excitation due au passage du train, on dispose d'un code de calcul bidimensionnel à déformation plane qui, à partir de la transformée de Fourier $\mathrm{P}(\mathrm{f})$ d'un signal linéique $\mathrm{p}(\mathrm{t})$, calcule la transformée de fourier $\mathrm{A}(\mathrm{f})$ de l'accélération linéique $\mathrm{a}(\mathrm{t})$ au point $\mathrm{B}$ grâce à la fonction de transfert $H(f)$ :

$$
\begin{gathered}
A(f)=H(f) \cdot P(f) \\
a(t)=h(t) * p(t)=\int_{-\infty}^{+\infty} h(\tau) p(t-\tau) d \tau
\end{gathered}
$$

Par résolution du problème inverse, la connaissance de $\mathrm{a}(\mathrm{t})$ conduit à la connaissance de l'excitation $\mathrm{p}(\mathrm{t})$. Afin d'appliquer cette démarche à la détermination de l'excitation due au train, il est nécessaire de satisfaire les hypothèses de base du modèle de calcul:

- linéarité: on admet qu'il n'y a pas d'interaction entre les fréquences contenues dans le signal, d'où la possibilité de décomposition et de recomposition par la transformation de Fourier;

- bidimensionnalité: le problème n'étant pas réellement bidimensionnel, il est nécessaire de recourir à certaines hypothèses simplificatrices ou/et à des calculs auxiliaires pour déduire de la fonction de transfert bidimensionnelle la fonction de transfert tridimensionnelle.

- Une première méthode consiste à approcher, pendant le passage du train, le signal induit au niveau des rails par la même fonction $\mathrm{u}(\mathrm{t})$ en tout point. Dans la zone considérée, de dimension petite par rapport à la longueur du train, le problème est alors bidimensionnel.

- Une deuxième méthode, que nous avons adoptée, consiste à supposer que chaque point $M$ des rails, d'abscisse $x$, subit la même excitation mais avec un certain déphasage $\mathrm{x} / \mathrm{V}$.

$$
u(x, t)=u\left(t-\frac{x}{V}\right)
$$

où $\mathrm{V}$ est la vitesse du train.

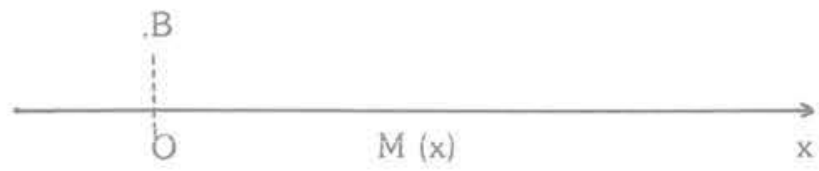

Le problème est alors tridimensionnel et si nous appelons

$v(x, t)$ le signal au point $B$ du à l'excitation en $M$, $V(f, x)$ sa transformée de Fourier,

$\mathrm{U}(\mathrm{f})$ la transformée de Fourier de $\mathrm{u}(\mathrm{t})$ et $H(f, x)$ la fonction de transfert entre $M$ et $B$,

alors

$$
V(f, x)=H(f, x) U(f) \exp (-2 i \pi f x / V)
$$



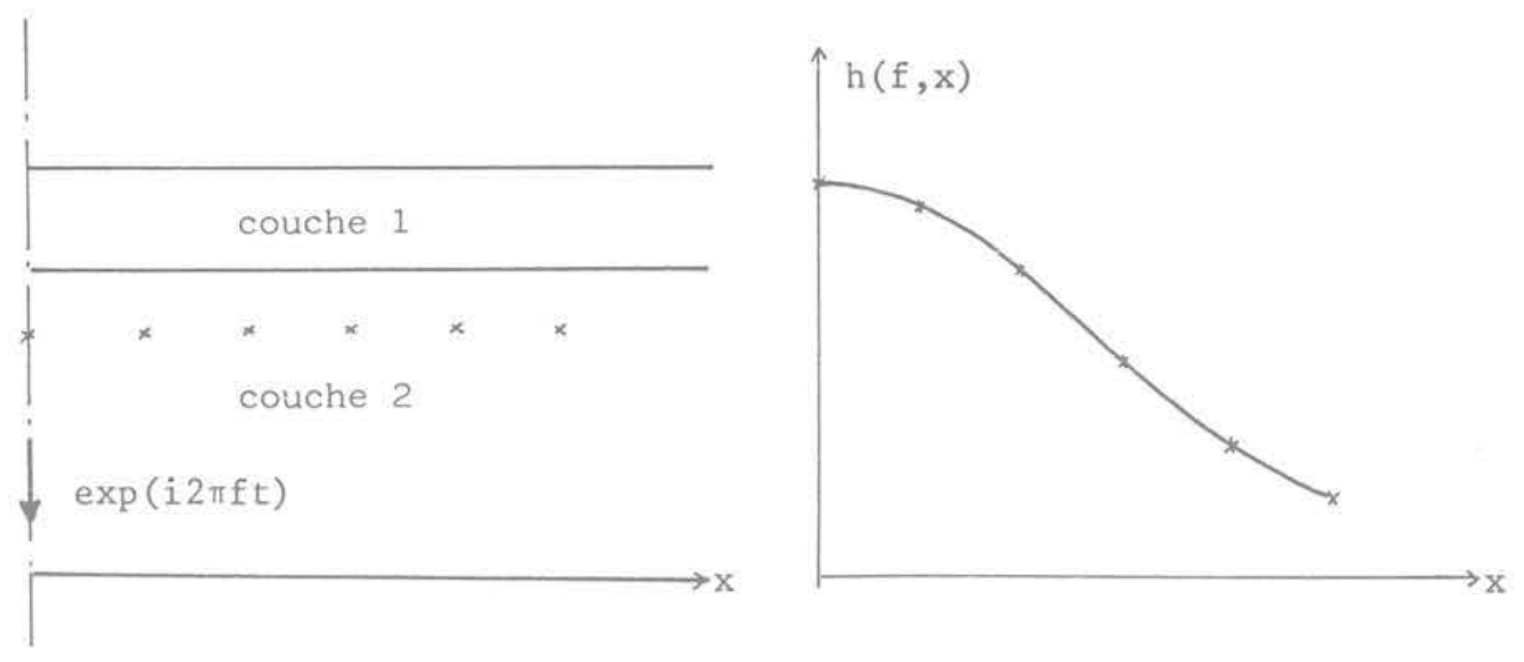

Fig. 5. - Détermination de la forme de la fonction de transfert $H(f, x)$.

Si nous appelons $w(t)$ le signal au point $B$ du au passage du train et $W(f)$ sa transformée de Fourier,

$$
W(f)=\left[\int_{-\infty}^{+\infty} H(f, x) \exp (-2 i \pi f x / V) d x\right] U(f)
$$

Or, nous disposons par le biais du calcul bidimensionnel de la fonction de transfert $\eta$ (f)

$$
\eta(f)=\int_{-\infty}^{+\infty} H(f, x) d x
$$

dont nous aimerions déduire la fonction de transfert

$$
\kappa(f)=\int_{-\infty}^{+\infty} H(f, x) \exp (-2 i \pi f x / V) d x
$$

obtenue par l'expérience in situ.

Pour calculer $\kappa(\mathrm{f})$, nous définissons un problème auxiliaire axisymétrique qui donnera une approximation de la forme de la fonction $H(f, x)$ en fonction de $x$ : on considère le demi espace hétérogène de mêmes propriétés que le site du tunnel (qui respecte donc la symétrie de révolution) et on applique sur l'axe de symétrie une force d'amplitude unité et de fréquence f, à une profondeur égale à celle des rails sur le site. L'amplitude de la réponse $h(f, x)$ en un point de même profondeur que le point $\mathrm{B}$ et distant de $\mathrm{x}$ de l'axe de symétrie, est proche de la fonction de transfert $\mathrm{H}(\mathrm{f}, \mathrm{x})$. On peut supposer, en première approximation qu'elle en respecte la forme en fonction de $\mathrm{x}$.

$$
H(f, x)=A h(f, x) ; A \text { constante }
$$

Connaissant la forme de $\mathrm{H}(\mathrm{f}, \mathrm{x})$ et ayant calculé

$$
\eta(f)=\int_{-\infty}^{+\infty} H(f, x) d x=A \int_{-\infty}^{+\infty} h(f, x) d x
$$

on peut facilement déduire $\mathrm{A}$ et la fonction de transfert réelle

$$
\kappa(f)=\int_{-\infty}^{+\infty} H(f, x) \exp (-2 i \pi f x / V) d x
$$

qui ouvre la voie â la résolution du problème inverse:

$$
U(f)=W(f) / \kappa(f)
$$

\section{RÉALISATION PRATIQUE DE LA VALI- DATION DU CODE DE CALCUL [4]}

La validation du code de calcul a été réalisée sur un site se trouvant à Paris près de la station Porte d'Auteuil.

\subsection{Conditions des essais}

Les essais sismiques ont permis d'interpréter le sol à l'aide d'un modèle tricouche comportant une couche de remblai, une couche de marnes et calcaires remaniés avec inclusions de silex, une couche de craie sous la nappe (fig. 6).

La zone de sol traitée par injections, située autour du tunnel a dô être dotée de propriétés mécaniques différentes de celles du calcaire environnant. Les propriétés des matériaux en présence ont été réunies dans le tableau I; elles résultent des calculs effectués à partir du profil sismique brut présenté à la figure 7 .

Le calcul a été conduit avec une excitation harmonique verticale située sur l'axe du tunnel, d'où la possibilité de réduire les maillages de moitié, pour des raisons de symétrie. Nous avons parcouru le domaine de fréquences $10-100 \mathrm{~Hz}$ avec un pas de $10 \mathrm{~Hz}$. Pour réaliser un compromis optimum entre la précision du calcul (taille de la maille inférieure a $\lambda_{s} / 8$, si $\lambda_{s}$ est la longueur d'onde des ondes de cisaillement) et son cout, nous avons choisi un maillage pour les fréquences 10 à $40 \mathrm{~Hz}$ et un autre maillage, plus fin, pour les fréquences 50 à $100 \mathrm{~Hz}$. 


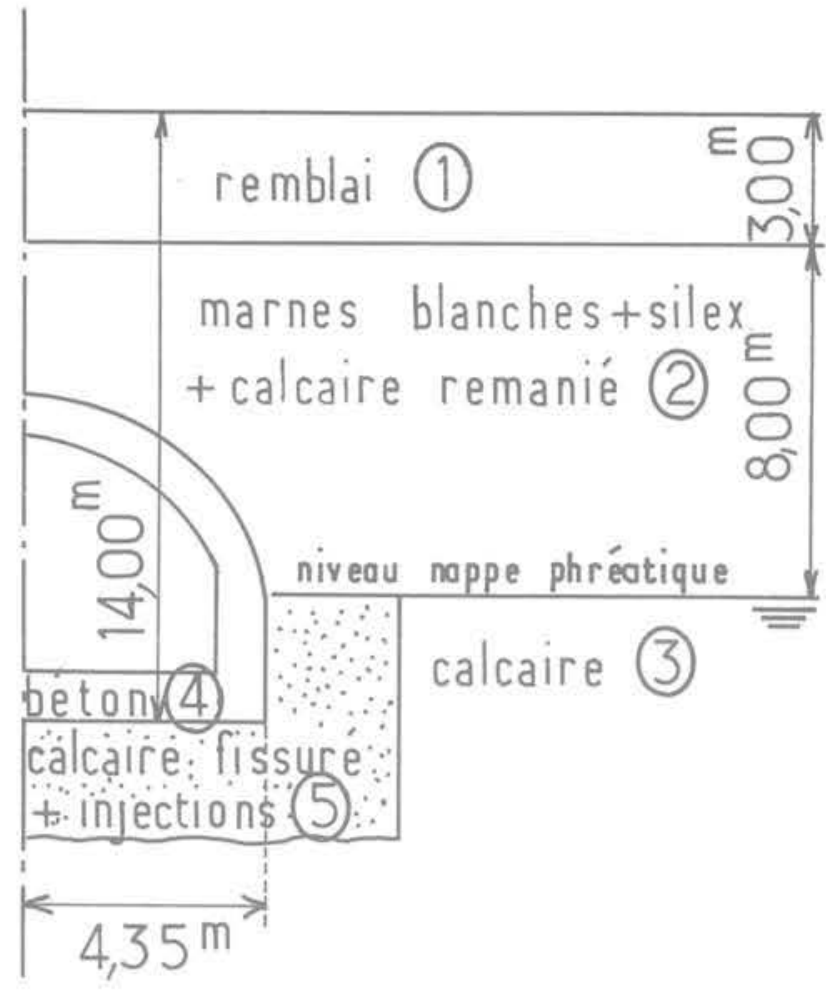

Fig. 6. - Modèle tricouche du site de la Porte d'Auteuil.

Les essais harmoniques ont été réalisés aux mêmes fréquences, l'enregistrement du champ vibratoire induit se faisant à partir d'accélomètres piézoélectriques fixés en différents points du tunnel (radier, piedroits et toit) et du sol (en surface et dans des sondages).

La comparaison essai-calcul a ainsi été faite par confrontation des courbes de réponse calculées et des courbes de réponse mesurées en différents points du modèle (points C, E, N, G de la figure 6), mais aussi par le calage des profils horizontaux calculés «amplitude-distance à l'axe du tunnel» sur les quelques points mesurés de ces mêmes profils.

\subsection{Courbes de réponse}

L'étalonnage des courbes de réponse calculées sur les courbes de réponse mesurées est subordonné d'abord au choix des modules des différents matériaux (étalonnage des fréquences de résonance, étalonnage grossier des amplitudes) et ensuite au choix de l'amortissement (qui conditionne un étalonnage plus fin des amplitudes); d'où la dualité des courbes calculées présentées sur la fig. 8 correspondant à un calcul sans amortissement (cf. tableau I) et à un calcul avec amortissement $(\beta=5 \%$ pour les matériaux $1,2,3,5$ du tableau I).

\subsubsection{Modèle sans amortissement}

Dans le tunnel (point C), calculs et essais se rejoignent pour mettre en évidence une résonance peu marquée à $40 \mathrm{~Hz}$ et une autre plus marquée à $90 \mathrm{~Hz}$. Les amplitudes calculées étant plus faibles que les ampli-

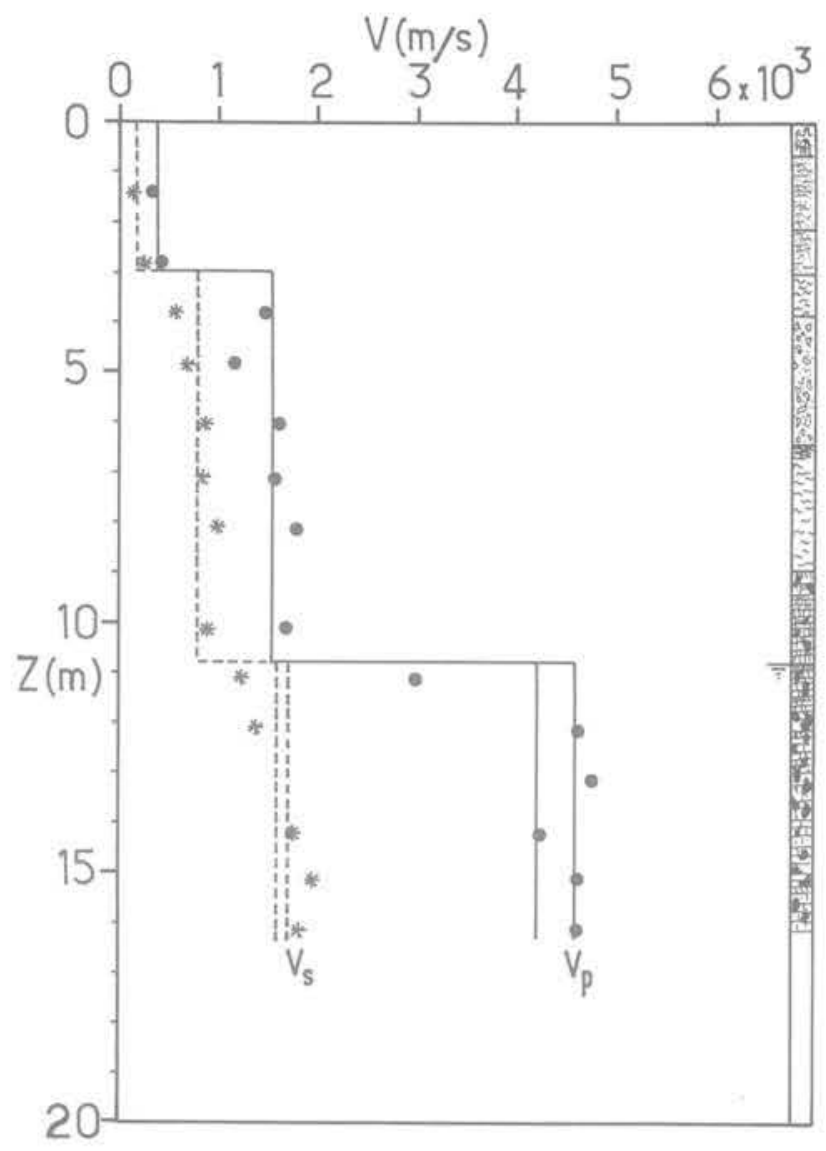

Fig. 7. - Profils des vitesses sismiques et coupe lithologique; interprétation par un modèle tricouche.

Vitesses de cisaillement mesurées Vitesses de compression mesurées

-. Vitesses de cisaillement moyenne par couche

- Vitesses de compression moyenne par couche.

tudes des mesures, une diminution du module du béton serait souhaitable.

Dans le sol (points E, N, G), les fonctions de transfert calculés sont de façon générale moins amorties que leurs analogues mesurées:

- Au niveau du radier (point E), il n'y a pas de résonance expérimentale franche mais un faible maximum relatif apparaît vers $20 \mathrm{~Hz}$ et un autre plus net à $90 \mathrm{~Hz}$. Le pic à $40 \mathrm{~Hz}$ prévu par le calcul n'est pas décelable expérimentalement. Les amplitudes calculées et les amplitudes mesurées sont comparables.

- A la surface du sol (point $\mathrm{N}$ ), le comportement vibratoire expérimental peut être raisonnablement prévu par le calcul jusqu'à $60 \mathrm{~Hz}$ (faible résonance à $40 \mathrm{~Hz}$ ) mais les écarts essais-calculs deviennent importants au-delà de $60 \mathrm{~Hz}$ (résonance calcul à $100 \mathrm{~Hz}$ trop marquée).

- Au niveau des piedroits (point G), essais et calculs donnent une évolution parallèle jusqu'à $60 \mathrm{~Hz}$ avec cependant surestimation par les essais par rapport aux 
Tableau 1. - Propriétés dynamiques des matériaux en présence

\begin{tabular}{|c|c|c|c|c|c|}
\hline Type de matériau & $\begin{array}{l}\text { Numéro } \\
\text { de } \\
\text { matériau }\end{array}$ & $\begin{array}{l}\text { Module de } \\
\text { cisaillement } \\
\text { G (MPa) }\end{array}$ & $\begin{array}{c}\text { Coefficient } \\
\text { de Poisson } \\
\text { V }\end{array}$ & $\begin{array}{c}\text { Amortissement } \\
\beta(\%)\end{array}$ & $\begin{array}{c}\text { Masse } \\
\text { volumique } \\
\rho\left(\mathrm{kg} / \mathrm{m}^{3}\right)\end{array}$ \\
\hline 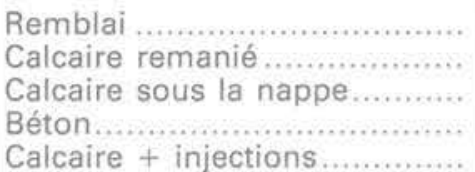 & $\begin{array}{l}1 \\
2 \\
3 \\
4 \\
5\end{array}$ & $\begin{array}{r}67 \\
1293 \\
5670 \\
12600 \\
3500\end{array}$ & $\begin{array}{l}0,370 \\
0,315 \\
0,420 \\
0,160 \\
0,300\end{array}$ & $\begin{array}{l}0 \\
0 \\
0 \\
0 \\
0\end{array}$ & $\begin{array}{ll}2 & 000 \\
2 & 020 \\
2 & 100 \\
2 & 540 \\
2 & 675\end{array}$ \\
\hline
\end{tabular}
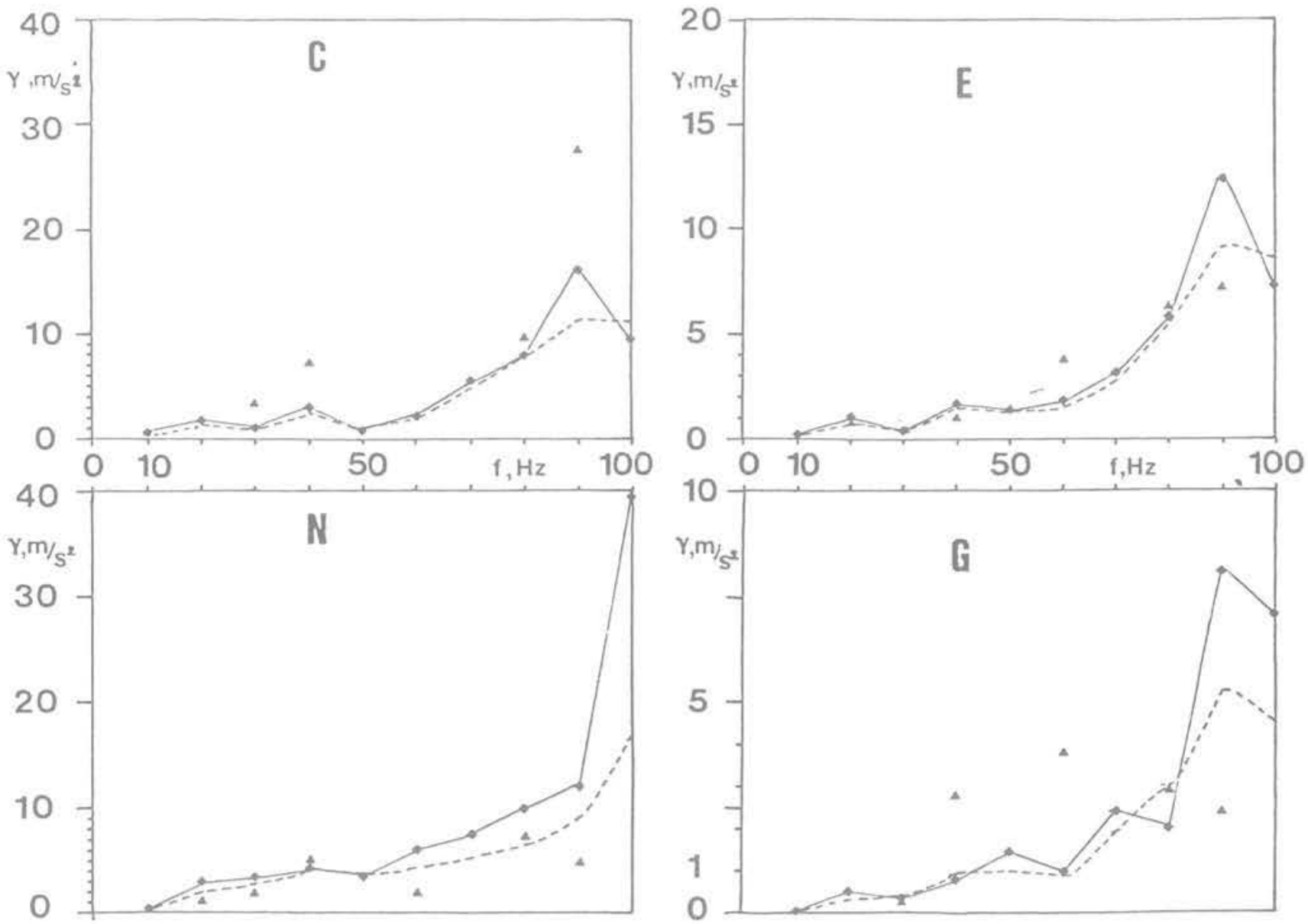

Fig. 8. - Fonctions de transfert en accélérations verticales (excitation: $10^{6} \mathrm{~N} / \mathrm{ml}$ ) en différents points de mesure:

A Mesures interprétées en bidimensionnel

Calculs sans amortissements

Calculs avec amortissements $(\beta=5 \%)$.

calculs; au-delà de $60 \mathrm{~Hz}$, la résonance à $90 \mathrm{~Hz}$ prévue par le calcul n'est absolument pas vérifiée expérimentalement.

\subsubsection{Modèle avec amortissement}

L'amortissement visqueux n'agit pratiquement pas sur les valeurs des fréquences de résonance mais il influe notablement sur les valeurs des amplitudes aux fré- quences de résonance, surtout lorsque ces résonances sont très marquées $(90 \mathrm{~Hz})$.

Sur le tunnel, le spectre calculé avec amortissement est trop étalé par rapport au spectre mesuré.

Dans le sol, la prise en compte d'un amortissement est très favorable à une meilleure concordance essaiscalculs (point E par exemple). 


\subsubsection{Conclusion}

Il $y$ a globalement un bon accord entre essais et calculs (sauf très localement) à condition d'ajuster les modules: la non linéarité du sol justifie, par exemple, de choisir un module deux fois plus petit que celui qu'il a été en $\mathrm{N}$, au-delà de $60 \mathrm{~Hz}$, à cause de l'ordre de grandeur important des déformations et de l'allure décroissante des courbes module-déformation [2]; mais la diminution du module conduisant à une augmentation trop considérable des amplitudes, un amortissement plus grand deura être choisi pour compenser cette tendance.

\section{UTILISATION DU CODE DE CALCUL POUR LA MISE EN ÉVIDENCE DES PA- RAMẼTRES ESSENTIELS DU PROBLĖME}

A côtế des paramètres propres au tunnel (formes, dimensions, profondeur) ou de paramètres artificiels destinés à atténuer les vibrations (revêtement amortissant, écran isolant, tranchées, etc.), ce sont essentiellement - comme le montre bien le calcul - les paramètres caractéristiques du sol qui jouent un rôle primordial dans les phénomènes de génération et de propagation des vibrations dues au métro: c'est ce que nous allons voir dans ce paragraphe en examinant l'effet de différents paramètres sur les courbes de réponse en surface du sol (point $\mathrm{N}$ défini plus haut).
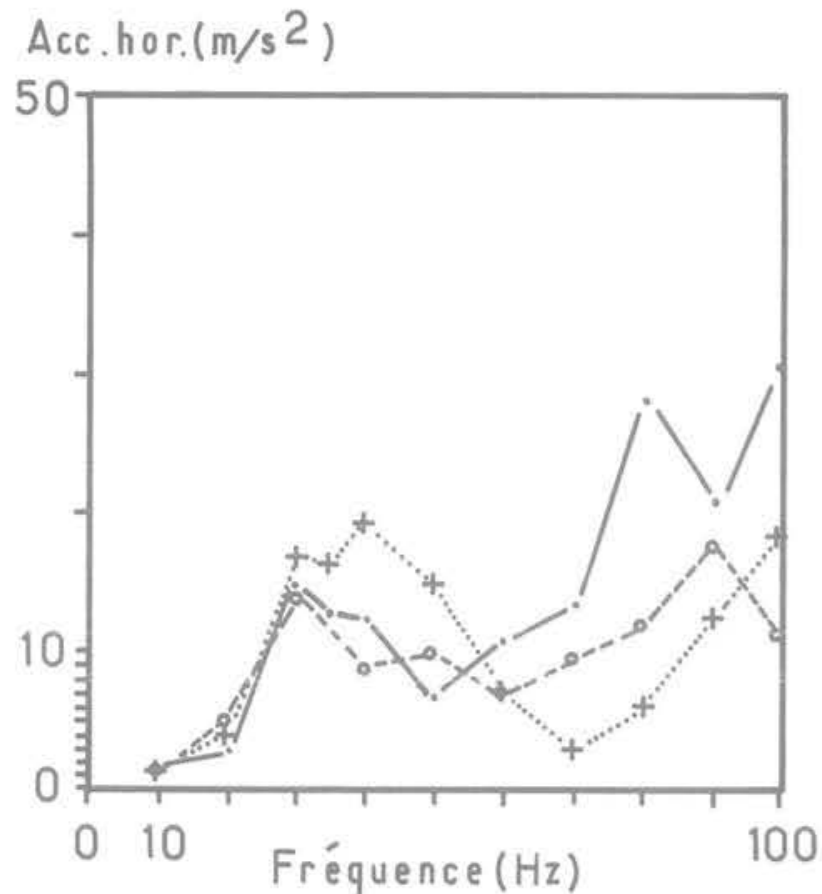

\subsection{Paramètres de la loi rhéologique}

Comme nous l'avons déjà entrevu lors de l'étalonnage des courbes de réponse calculées sur les courbes mesurées, la connaissance de la raideur et de l'amortis. sement est essentielle; le coefficient de Poisson présente une moindre importance.

\subsubsection{Influence de la raideur (module)}

Lorsque le module de cisaillement $\mathrm{G}$ augmente (voir fig. 9), le contenu en fréquences tend vers une répartition plus uniforme comme si, globalement, on augmentait les impédances complexes (amortissement global) d'interaction sol-tunnel. Sachant que le calcul surestime les fréquences élevées (voir \$2.2) et que certaines résonances sont dues aux réflexions sur le toit du tunnel (entrée en résonance de la zone de sol située au-dessus du tunnel, vers $80 \mathrm{~Hz}$, pour $\mathrm{G}=$ $200 \mathrm{MPa}$ ), nous considérerons que les phénomènes d'interaction tunnel-sol se manifestent dans le domaine $0-70 \mathrm{~Hz}$. Sur cette plage de fréquences, l'effet de Paugmentation de G, tout à fait équivalent en accélérations verticales et en accélérations horizontales est « un effet de rigidification " se traduisant par une augmentation des fréquences de résonance (voir tabl. II); la diminution des amplitudes à la résonance que l'on attendrait normalement est ici occultée à cause de l'augmentation du coefficient d'amortissement.

Ainsi, toute méthode d'amélioration du sol visant à augmenter le module sera à conseiller:

- Le compactage peut être une solution appropriée dans la mesure où il s'applique non seulement au sol

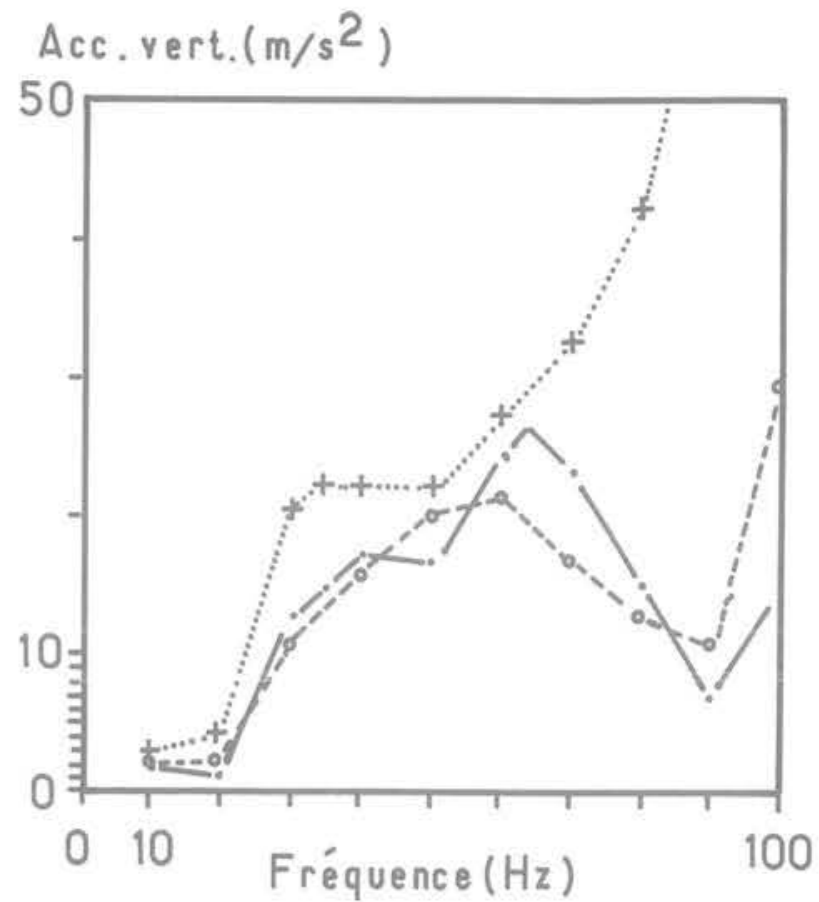

Fig. 9. - Comparaison des courbes de réponse en surface du sol, en accélération horizontales (a) et verticales (b) pour une excitation linéique sur le radier de $10^{6} \mathrm{~N} / \mathrm{ml}$.

$$
\begin{aligned}
& . \ldots+\ldots-. \mathrm{G}=200 \mathrm{MPa}, \beta=5 \% \\
& +\ldots+\ldots+\ldots \mathrm{G}=400 \mathrm{MPa}, \beta=3 \% \\
& 0 \ldots \ldots \mathrm{G}=4000 \mathrm{MPa}, \beta=1 \% .
\end{aligned}
$$


Tableau II. Influence de I'augmentation du module de cisaillement sur les courbes de réponse en accélérations verticales

\begin{tabular}{|c|c|c|c|}
\hline $\begin{array}{c}\mathrm{G} \\
(\mathrm{MPa})\end{array}$ & $\begin{array}{c}\beta \\
(\%)\end{array}$ & $\begin{array}{c}\text { Fréq. } \\
(\mathrm{Hz})\end{array}$ & $\begin{array}{c}\text { Ampl. } \\
\left(\mathrm{m} / \mathrm{s}^{2}\right)\end{array}$ \\
\hline 200 & 5 & 40 & 17 \\
400 & 3 & 50 & 23 \\
4000 & 1 & 70 & 10 \\
\hline
\end{tabular}

de surface mais aussi au sol de fondation du tunnel (en profondeur) et que l'augmentation du module qui en découle soit suffisante (ce qui est possible si le modèle de départ est faible): dans la pratique, ce sera le cas pour les tunnels construits à fouille ouverte et dont la fouille est ensuite remblayée.

- L'injection de matériaux spéciaux rigidifiants autour du tunnel, si elle provoque la rigidification d'une zone suffisamment étendue enserrant le tunnel, peut être bénéfique.

\subsubsection{Influence de Pamortissement}

Comme nous l'avons vu lors de l'étalonnage du code de calcul, l'amortissement agit peu sur le contenu en fréquences (voir fig. 8) mais il affecte beaucoup les amplitudes de résonance (voir fig. 9).

Il s'ensuit que toute méthode d'amélioration du sol visant à augmenter l'amortissement est à conseiller en

$$
\text { Acc.hor. } \mathrm{m} / \mathrm{s}^{2}
$$

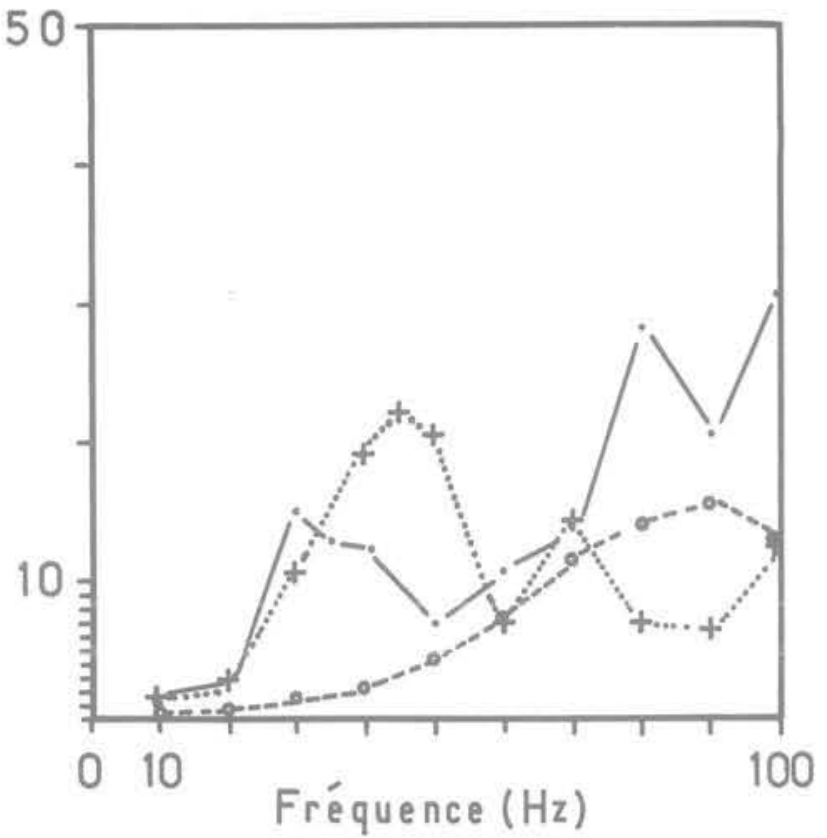

cas de résonances très marquées: hétérogénéités locales, injection de matériaux amortissants, etc.

\subsubsection{Influence du coefficient de Poisson}

Contrairement au module et à l'amortissement, le coefficient de Poisson a une action assez faible sur les courbes de réponse (cf. fig. 10: $v=0,30$ et $v=$ 0,485 ). Jusqu'à $60 \mathrm{~Hz}$, l'effet est quasiment nul. Au-delà de $60 \mathrm{~Hz}$, la variation de v n'intervient pas en tant que cause intrinsèque d'une évolution quelconque des courbes de réponse mais plutôt en tant que cause indirecte conduisant à l'établissement d'hétérogénéités dans le sol (voir $\S 3.2 .5$ ).

\subsection{Influence des hétérogénéités}

3.2.1. L'homogénéisation d'un milieu stratifié tel que celui qui représente le site de la Porte d'Auteuil (cf. fig. 6), c'est-à-dire le remplacement de ce milieu hétérogène par un milieu homogène de propriétés mécaniques "équivalentes", est un procédé de calcul simplificateur très courant en mécanique des sols. La figure 11 présente les courbes de réponse en surface (point $\mathrm{N}$ ) du modèle tricouche et du modèle homogène équivalent, en supposant que les propriétés mécaniques équivalentes résultent de la moyenne des propriétés des trois couches:

$\mathrm{G}=794 \mathrm{MPa} \quad v=0,355 \quad \rho=2000 \mathrm{Kg} / \mathrm{m}^{3}$

L'homogénéisation tend à favoriser l'émergence d'une résonance très marquée, surtout en accéléra-

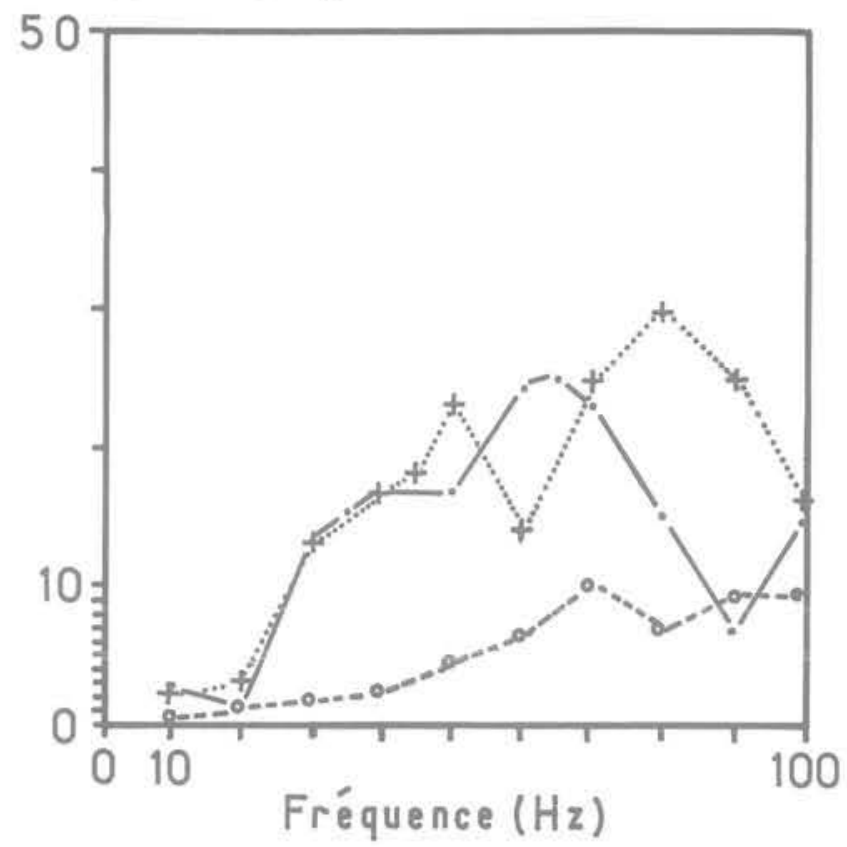

Fig. 10. - Comparaison des courbes de réponse en surface du sol, en accélérations horizontales (a) et verticales (b) pour une excitation linéique verticale sur le radier de $10^{6} \mathrm{~N} / \mathrm{ml}$. $-. . .-v=0,3$ gravier sec

$+\ldots+\ldots$ sol bicouche: graviers avec nappe phréatique sous le radier ○ _.. o _. o $v=0,485$ gravier saturé, nappe phréatique au-dessus du tunnel. 

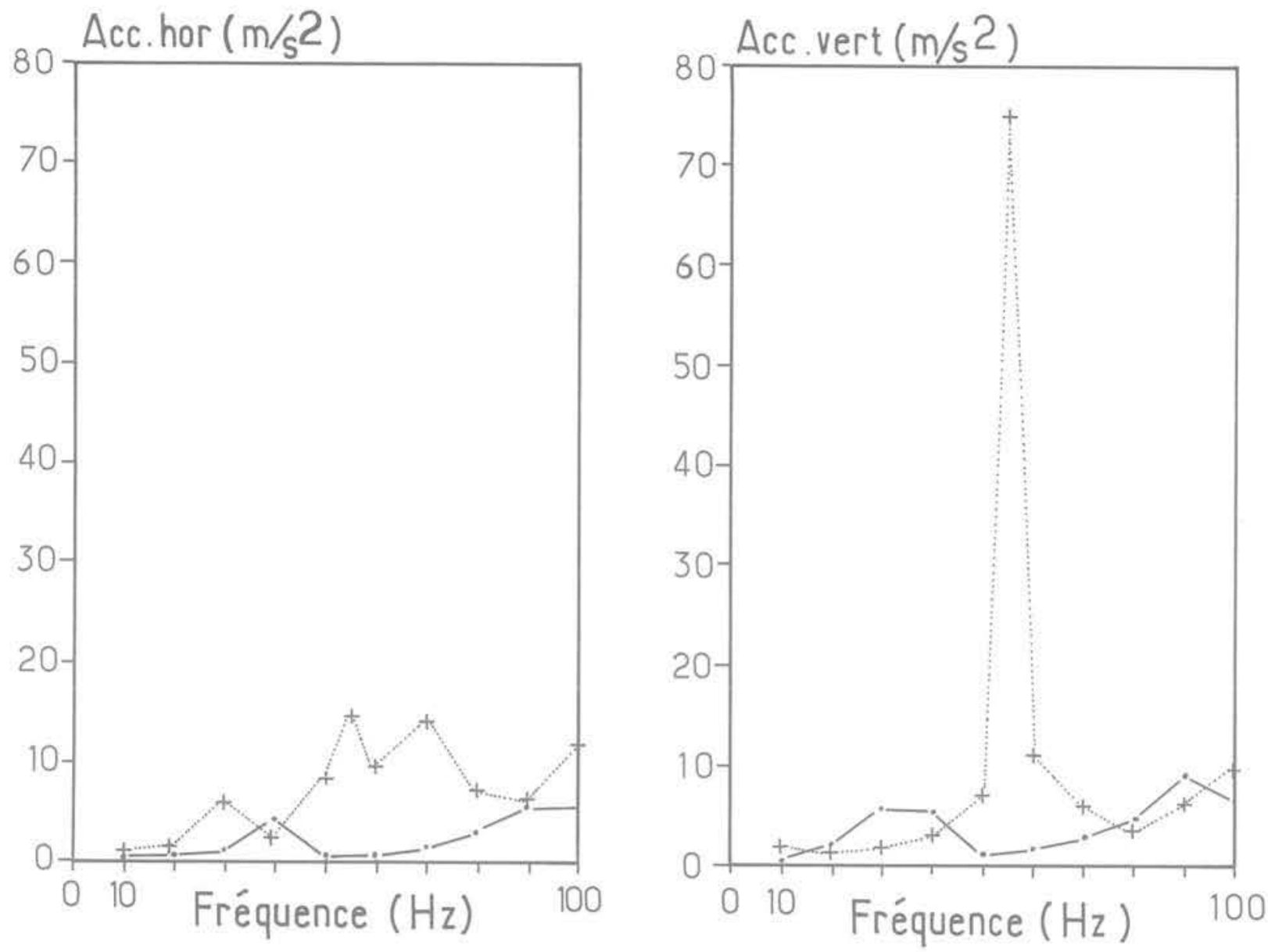

Fig. 11. - Comparaison des courbes de réponse en surface du sol, en accélérations horizontales (a) et verticales (b) pour une excitation verticale linéique sur le radier du tunnel de $10^{6} \mathrm{~N} / \mathrm{ml}$.

$$
+\ldots+\ldots+\ldots \text { Modèle homogène équivalent. }
$$

tions verticales (cf. fig. 11) à $55 \mathrm{~Hz}$. Mais les fréquences de couches sont systématiquement occultées.

Dans le cas présent d'une source enterrée, il faut donc envisager l'homogénéisation avec beaucoup de prudence, l'équivalence modèle hétérogène - modèle homogène loin de la source n'étant pas toujours assurée de façon satisfaisante.

3.2.2. Le substratum rigide influe d'abord sur la fréquence de résonance d'interaction du système tunnel-sol comme un agent rigidifiant de ce système à mesure que le substratum est plus «lié » au tunnel. La figure 12 montre bien l'augmentation des fréquences de résonance et la diminution simultanée des amplitudes calculées, en accélérations, à la surface du sol: voir tabl. III.

Un autre effet de la présence du substratum est l'effet de réflecteur des ondes de compression et de cisaillement, avec amplification très forte à certaines fréquences, dites résonances de couche (ici $80 \mathrm{~Hz}$, dans le cas où le substratum sert de soubassement à une couche d'alluvions de $9 \mathrm{~m}$ ).
Tableau III. - Effet du substratum rigide: la réponse, résultante est une superposition de la réponse d'interaction sol-tunnel, de la réponse propre de la couche de matériau meuble située au-dessus du substratum et de la réponse propre de la zone de sol située au-dessus du tunnel. Les deux modes s'occultent parfois, d'où des résonances peu perceptibles.

\begin{tabular}{|c|c|c|c|c|}
\hline & \multicolumn{2}{|c|}{$\begin{array}{l}\text { Accélérations } \\
\text { horizontales }\end{array}$} & \multicolumn{2}{|c|}{$\begin{array}{c}\text { Accélérations } \\
\text { verticales }\end{array}$} \\
\hline & $\begin{array}{l}\text { Fréq. } \\
(\mathrm{Hz})\end{array}$ & $\begin{array}{l}\text { Ampl. } \\
\left(\mathrm{m} / \mathrm{s}^{2}\right)\end{array}$ & $\begin{array}{l}\text { Fréq. } \\
(\mathrm{Hz})\end{array}$ & $\begin{array}{l}\text { Ampl. } \\
\left(\mathrm{m} / \mathrm{s}^{2}\right)\end{array}$ \\
\hline $\begin{array}{l}\text { Substratum } \\
\text { très profond }\end{array}$ & 30 & 15 & 35 & 17,5 \\
\hline $\begin{array}{l}\text { Substratum } \\
\text { sans radier }\end{array}$ & 40 & 10 & $\begin{array}{c}50 \\
\text { (peu } \\
\text { percep- } \\
\text { tible) }\end{array}$ & 12,5 \\
\hline $\begin{array}{l}\text { Substratum au } \\
\text { dessus du toit }\end{array}$ & $\begin{array}{c}60 \\
\text { (peu } \\
\text { percep- } \\
\text { tible) }\end{array}$ & 7,5 & 70 & 10 \\
\hline
\end{tabular}



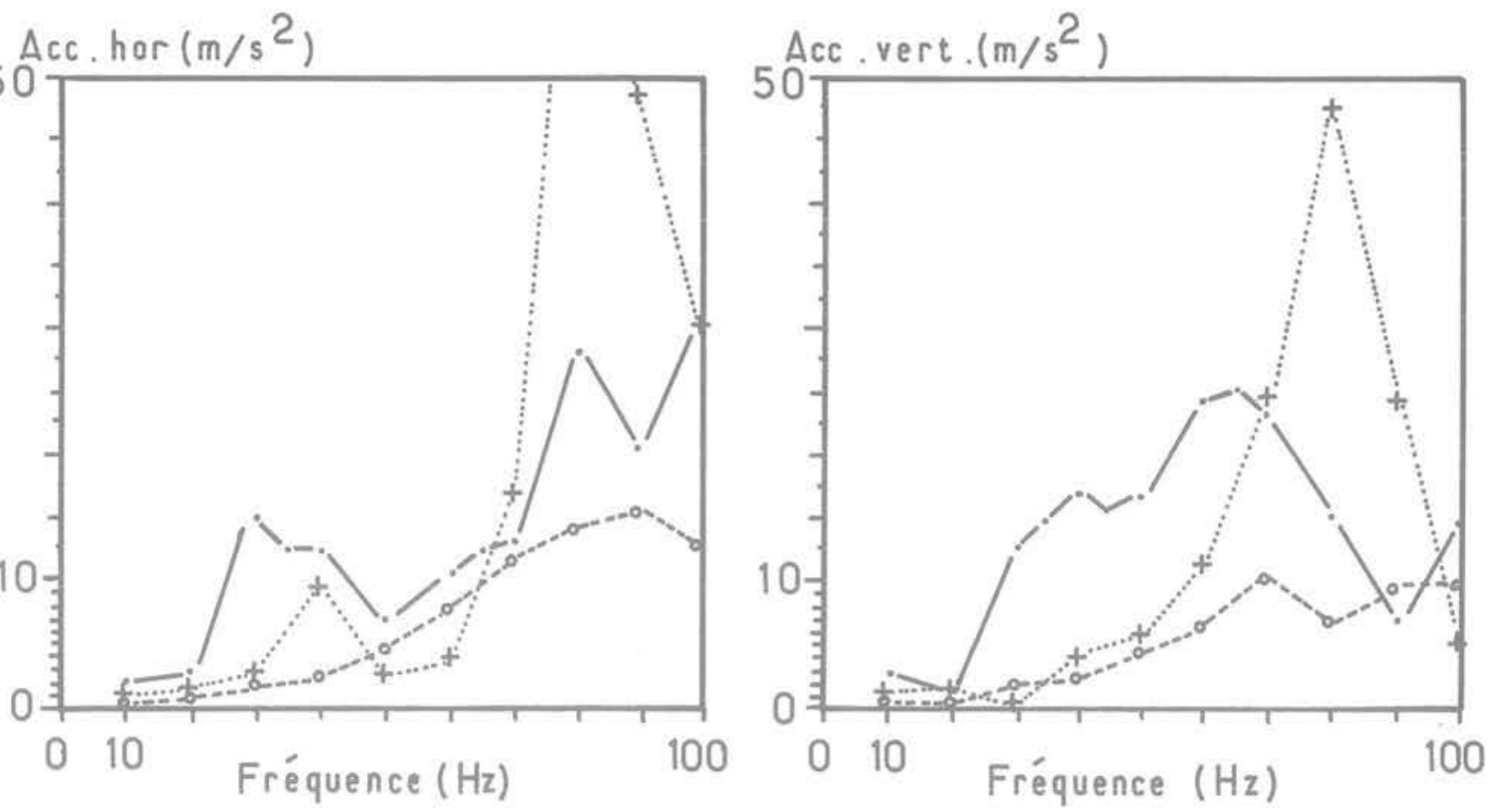

Fig. 12. - Comparaison des courbes de réponse en surface du sol, en accélérations horizontales (a) et verticales (b) pour une excitation verticale linéique sur le radier du tunnel de $10^{6} \mathrm{~N} / \mathrm{ml}$. .-....- Pas de substratum (couche d'alluvions semi-infinie) $+\ldots+\ldots+\ldots$ Substratum au niveau du radier (couche d'alluvion de $9 \mathrm{~m}$ ) o _._. o . . . Substratum affleurant (tunnel dans le substratum).

La présence d'un substratum rigide sera donc un élément à surveiller avec beaucoup d'attention en fonction de sa position par rapport à celle du tunnel et en fonction des résonances de couches auxquelles il peut donner lieu. Dans la pratique, il est toujours favorable d'implanter le tunnel dans le substratum lui-même.

3.2.3. La position de la nappe phréatique (définie comme milieu à coefficient de Poisson élevé) agit assez peu sur l'interaction sol-tunnel comme le montre bien la figure 10, pour les fréquences inférieures à $60 \mathrm{~Hz}$. En revanche, la nappe peut jouer le rôle de réflecteur d'ondes de compression (voir fig. 10b), avec des résonances de couches très marquées en accélérations verticales. La remontée de la nappe sera donc un paramètre à surveiller essentiellement de ce point de vue et non du strict point de vue de la transformation des propriétés qu'elle entraîne.

\section{CONCLUSION}

En résumé, on obtient une bonne concordance essaiscalculs si l'on accepte le préalable indispensable d'une connaissance satisfaisante des propriétés des différents matériaux en présence et, de manière prépondérante, du sol.

$\mathrm{Ce}$ dernier peut être entièrement défini pour le problème qui nous intéresse par des essais sismiques in situ de type «cross hole» complétés par des essais de laboratoire (cycliques ou de colonne résonante).
L'outil de calcul, ainsi mis au point et validé, conduit à de larges possibilités d'études paramétriques de prévention pour le comportement vibratoire des tunnels et, donc, de test pour les méthodes curatives du problème de la transmission des vibrations du métro au milieu environnant.

\section{BIBLIOGRAPHIE}

1. HOAR, R.J.; STOKOE, K.J. (1981). - Cross hole measurement and analysis of shear waves. Compte rendu du $\mathrm{X}^{\mathrm{e}}$ congrès international de Mécanique des Sols, vol. 3, pp. 223-226, Stockholm, 1981.

2. EL HOSRI, M.S. (1980), - Contribution à l'étude des propriétés mécaniques des matériaux en régime non permanent. Thèse présentée à l'Ecole Centrale des Arts et Manufactures pour obtenir le grade de Docteur Ingénieur.

3. RUCKER, W. (1980). - Ermittlung der Schwingungserregung beim Betrieb schienengebundener Fahrzeuge in Tunneln, sowie Untersuchung des Einflusses einzelner Parameter auf die Ausbreitung von Erschütterungen im Tunnel und dessen Umgebung. Forschungsbericht 64, B.A.M. Berlin, 1980.

4. LEFEBURE, D. (1979). - Mesure des propriétés rhéologiques du sol pour le calcul des fondations vibrantes; étude expérimentale in situ. Thèse présentée à l'Ecole Centrale des Arts et Manufactures pour obtenir le grade de Docteur Ingénieur. 